Open Access

\title{
Gastric Syphilis and Membranous Glomerulonephritis
}

\author{
Min Roh', Joo Hyun Sohn', Tae Yeob Kim¹, Sung Jong Kim¹, Ji Soong Kim¹, Sung Jun Chung1, Ju Yeon Pyo² \\ and Young-Ha Oh${ }^{2}$ \\ Departments of ${ }^{1}$ Internal Medicine and ${ }^{2}$ Pathology, Hanyang University Guri Hospital, Hanyang University College of Medicine, Guri, Korea
}

\begin{abstract}
Syphilis is a chronic systemic infectious disease caused by the bacterium Treponema pallidum. Gastric involvement and nephrotic syndrome are uncommon but well documented complications of syphilis, but the co-occurrence of these two complications in the same patient is extremely rare. Thus, because of their nonspecific presentation, suspicion of gastric syphilis (GS) and nephrotic syndrome is essential for diagnosis. Patients should be investigated thoroughly and a diagnosis made based on clinical, endoscopic, and histological findings, in order to initiate appropriate therapy. We report of a 34-year-old male patient with a history of epigastric pain and a diagnosis of GS and syphilis-associated membranous glomerulonephritis confirmed by gastroscopy and kidney biopsy, who was treated successfully with penicillin G benzathine. This case report provides information on the typical features of GS that should help raise awareness of this rare disease entity among clinicians, resulting in earlier diagnosis and administration of appropriate therapy.
\end{abstract}

Key Words: Glomerulonephritis, membranous; Stomach; Syphilis

\section{INTRODUCTION}

Syphilis is a sexually transmitted disease caused by infection with the bacterium Treponema pallidum whose diagnosis remains elusive. The introduction of penicillin approximately 60 years ago along with public health improvements led to a decline in syphilis infection rates. ${ }^{1}$ However, after the rise of acquired immunodeficiency syndrome, the incidence of reported cases of syphilis increased. ${ }^{2}$ Moreover, in South Korea, the number of reported cases of syphilis gradually increased from 134 to 1,548 between 2002 and 2008. ${ }^{3}$ Syphilis is clinically subdivided into three stages: primary, secondary, and tertiary. Secondary syphilis can spread to the stomach, kidney, liver, joints, and eyes. The first two cases of gastric syphilis (GS) were reported in 1834 by Andral, ${ }^{4}$ and the first histopathologically confirmed case of GS was reported by Graham at 1922 in a patient whose tissues had been surgically removed. ${ }^{4}$ Sub-

Received: March 4, 2014 Revised: May 2, 2014

Accepted: June 16, 2014

Correspondence: Joo Hyun Sohn

Department of Internal Medicine, Hanyang University Guri Hospital, Hanyang University College of Medicine, 153 Gyeongchun-ro, Guri 471-701, Korea Tel: +82-31-560-2177, Fax: +82-31-560-2598, E-mail: sonjh@hanyang.ac.kr

(C) This is an Open Access article distributed under the terms of the Creative Commons Attribution Non-Commercial License (http://creativecommons.org/ licenses/by-nc/3.0) which permits unrestricted non-commercial use, distribution, and reproduction in any medium, provided the original work is properly cited. sequently, additional studies have described a high incidence of GS diagnoses based on clinical, serological, and radiological evidences. ${ }^{1}$ Moreover, kidney involvement due to syphilis has been reported during secondary, latent, and tertiary syphilis. ${ }^{5}$ Although a number of nephritic syndrome case reports associated with secondary syphilis have previously been published, it is considered a rare presentation. ${ }^{6}$ Furthermore, some cases of syphilis-related nephropathy were reported in the 1940s and 1950s, but relatively little has been published in more recent times. In addition, the co-occurrence of GS and nephrotic syndrome in the same patient is extremely rare. Here we report a particular case of GS presenting with concomitant membranous glomerulonephritis (MGN). As cases such as this represent a diagnostic challenge, this report provides information on the typical features of GS that might be helpful for raising awareness of this rare disease entity among clinicians, facilitating its earlier diagnosis and appropriate therapy.

\section{CASE REPORT}

A 34-year-old unmarried man presented to our hospital on February 28, 2011 with nocturnal epigastric pain and mild febrile sensation lasting 3 weeks. He also had generalized edema and an inguinal rash. Physical examination on admission 
revealed a blood pressure of 110/70 $\mathrm{mm} \mathrm{Hg}$, heart rate of 82 beats per minute, respiration rate of 14 breaths per minute, body temperature of $36.8^{\circ} \mathrm{C}$, and $1+$ pitting edema below the knee. No oral or genital lesions were observed. He did not have any important personal, family, hospitalization, surgery, or other diseases history. His last sexual encounter was with a woman approximately 2 months previously.

The patient initially underwent an endoscopy for the evaluation of epigastric pain. Multiple ulcers and erosions, extending from the cardia to the pylorus, were observed on upper endoscopy (Fig. 1). The gastric mucosa was thickened and friable, and associated with the ulcer lesions. Histologically, the biopsied tissue revealed gastric ulcer and diffuse active gastritis with dense plasma cell infiltration, as well as variable numbers of lymphocytes and neutrophils. Although no endarteritis or endophlebitis were observed, owing to the superficial nature of biopsied tissue, silver staining was performed because of the suspicion of GS. Numerous spirochetes were detected within the lamina propria and between epithelial cells, confirming the diagnosis of GS. In addition, some Helicobacter organisms were also identified in the superficial mucus layer (Fig. 2).

Laboratory investigations revealed normal hemoglobin, hematocrit, white blood cell count, and liver function. The re- sults for human immunodeficiency virus serology and hepatitis diagnostic panel were negative. The speckled and skeleton patterns of antinuclear antibodies were detected at a titer of 1:80 and 1:320, respectively. Anti-neutrophil cytoplasmic antibodies and complement levels were normal. The Venereal Diseases Research Laboratory (VDRL) syphilis serology test was positive, with a titer of 1:256, and the fluorescent treponemal antibody absorption (FTA-abs) and T. pallidum latex agglutination tests were also positive.

Urinalysis revealed $3+$ protein without blood or red blood cells, and a 24-hour urine collection revealed $11.3 \mathrm{~g}$ of protein. Laboratory data showed blood urea nitrogen, creatinine, and albumin values of $19 \mathrm{mg} / \mathrm{dL}, 1.2 \mathrm{mg} / \mathrm{dL}$, and $1.8 \mathrm{~g} / \mathrm{dL}$, respectively. Serum total cholesterol and triglycerides were elevated, with values of $301 \mathrm{mg} / \mathrm{dL}$ and $202 \mathrm{mg} / \mathrm{dL}$, respectively. At this stage, we suspected nephrotic syndrome secondary to syphilis, and a diagnostic kidney biopsy was performed.

The renal biopsy revealed slight thickening of glomerular capillary walls with segmental subepithelial 'pores' on tangentially cut sections on silver staining, but the mesangium was not expanded. Weak granular immunoglobulin G (IgG) and C3 staining along the glomerular capillary walls was observed on immunofluorescence microscopy. However, IgA, IgM, C1q,
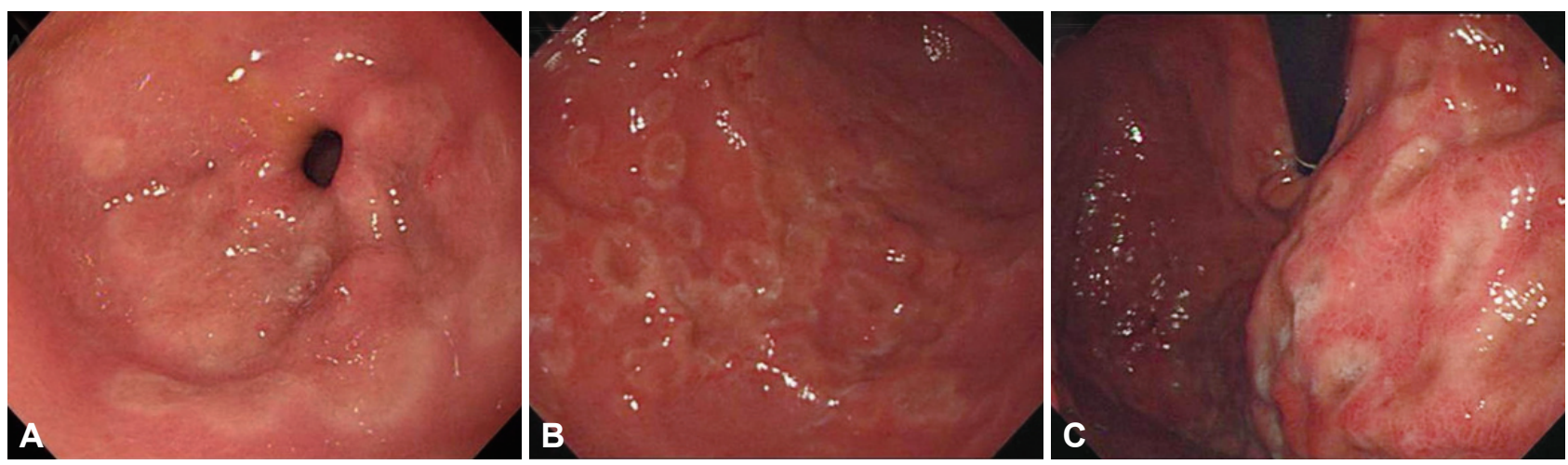

Fig. 1. Gastroscopic findings. Multiple irregular, shallow ulcers covered with whitish exudates and central depression in the (A) antrum, (B) body, and (C) cardia.
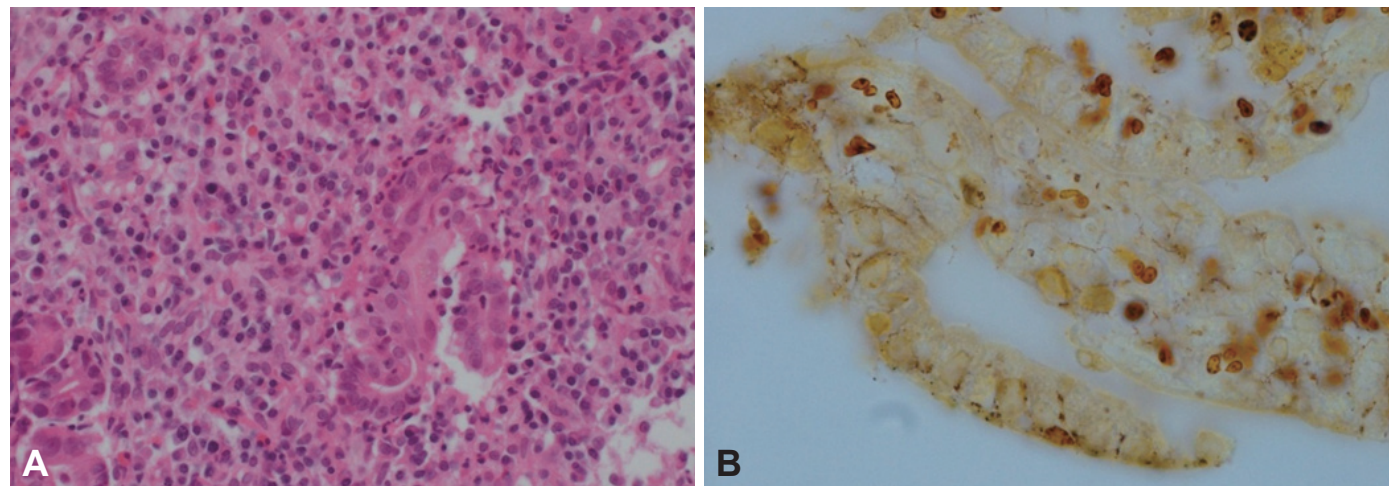

Fig. 2. Histologic findings of gastric biopsy. (A) Marked severe inflammation with lymphoplasmacytic infiltrates (H\&E stain, $\times 400)$. (B) Numerous spirochetes are present between foveolar epithelial cells (Warthin-Starry stain, $\times 1,000$ ). 

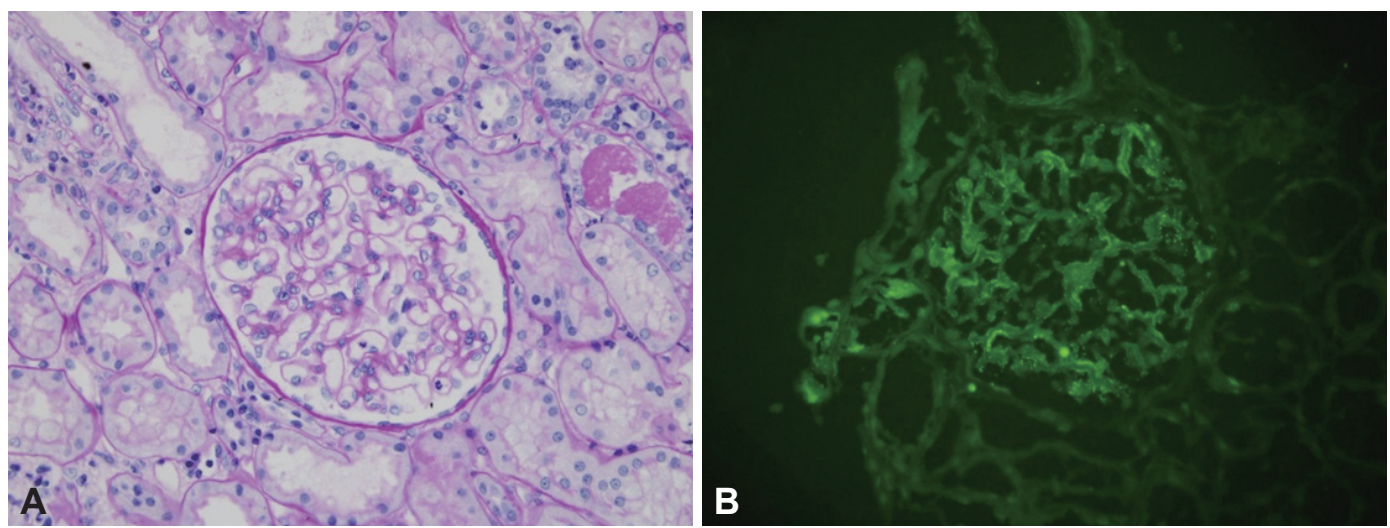

Fig. 3. Histologic findings of kidney biopsy. (A) Light microscopy showing slight thickening of the glomerular capillary walls (Periodic acidSchiff stain, $\times 400)$. (B) Immunofluorescence showing weak granular staining for immunoglobulin $\mathrm{G}$ along the capillary walls $(\times 400)$.

and C4 staining was negative. Electron microscopy (EM) could not be performed because the EM sample did not include the glomerulus (Fig. 3).

Correlation of clinical and serological findings with gastroscopy and renal biopsy results was consistent with GS and MGN secondary to syphilis. The patient was treated with 2.4 million units of penicillin $\mathrm{G}$ benzathine, leading to resolution of his clinical symptoms, including epigastric pain, skin rash, and generalized edema. The proteinuria also improved, with a reduction in the 24-hour urine protein values from 11.3 to $0.7 \mathrm{~g}$ after penicillin $\mathrm{G}$ treatment.

\section{DISCUSSION}

GS most commonly affects young adults in their second to fourth decade of life. Although GS patients generally experience epigastric pain, anorexia, early satiety, nausea, vomiting, and weight loss, ${ }^{7,8}$ these symptoms rarely contribute to GS diagnosis. ${ }^{1}$ A systematic review published in 2010 showed that the majority of patients with GS had no clinical history $(87 \%)$ or physical examination results (56\%) compatible with syphilis. Moreover, as no clear diagnostic criteria exist, establishing relevant associations between the medical, sexual history, and physical examination is important for diagnosis. Moreover, as symptoms are nonspecific, a high degree of diagnostic suspicion is especially required. As gastric involvement is usually seen after secondary syphilis when the spirochetes spread via the circulation, after considering the timing of past intercourse in our patient history, we estimated that he had secondary stage syphilis.

Different types of endoscopic findings have been reported in GS. Most patients show more than one lesion type, including multiple ulcerations (48\%), nodular mucosa (26\%), erosions (24\%), large ulcers (24\%), thickened folds (17\%), narrowing and rigidity (17\%), and mass lesions (2\%). ${ }^{1}$ Hypertrophy of the rugae at the antral and prepyloric regions is a common endoscopic finding. Moreover, rugal hypertrophy and poor distensibility may lead to misdiagnosis of scirrhous carcinoma or lymphoma. ${ }^{9}$ A reddish purple stomach color is thought to be characteristic of GS by some authors..$^{10}$ In our case, upper endoscopy revealed multiple irregular, shallow ulcers covered with whitish exudates and a central depression ranging from 0.5 to $2 \mathrm{~cm}$ in diameter in the antrum, body, and cardia, abruptly ending at the pyloric level. Complications including gastric hemorrhage, perforation, and gastric outlet obstruction have also been reported, but are less common. ${ }^{11}$ Spirochetes detection on silver staining is difficult in a background of elastic and reticulum fibers. Nevertheless, a positive finding of mucosal spirochetes on silver staining is considered an important diagnostic tool for GS. In our case, the patient was diagnosed with GS after a positive finding on silver staining.

Histopathological analysis findings compatible with endovasculitis include arterial wall and submucosal layer thickening, perivascular cell infiltrate, diffuse lymphocytes, and plasmocytes infiltrate. Although vasculitis, manifested by endarteritis or endophlebitis, is a typical finding in other sites, it is rarely observed in gastric samples, probably because endoscopic biopsies do not reach the submucosal layer.

The nephrotic syndrome that occurs in patients with syphilis is the most common clinical sign that occurs in $0.3 \%$ of patients with syphilis, ${ }^{12}$ and results from the growth of syphilis bacteria in the kidney. The mechanism of renal injury associated with syphilis involves an immune complex-mediated glomerulonephropathy, with MGN being the most common. However, in Korea, only one case of concurrent GS and nephrotic syndrome was reported by Kwon et al. ${ }^{13}$ in 2004.

In our case, the patient was diagnosed by using serology, gastroscopy, and renal biopsy, and his clinical symptoms and laboratory findings completely improved after penicillin treatment. Even though GS and MGN caused by syphilis are rare, it is important to emphasize this particular case owing to its diagnostic difficulty. Furthermore, as syphilis prevalence has 
seen a recent increase, it is likely that more patients will present to the clinic. Thus, recognition of the clinical picture of syphilis and its gastric and renal complications is important. By providing information on the typical features of GS, this case report will help to raise awareness of this rare disease entity among clinicians, resulting in earlier diagnosis and appropriate therapy.

\section{Conflicts of Interest}

The authors have no financial conflicts of interest.

\section{REFERENCES}

1. Mylona EE, Baraboutis IG, Papastamopoulos V, et al. Gastric syphilis: a systematic review of published cases of the last 50 years. Sex Transm Dis 2010;37:177-183.

2. Hook EW 3rd, Marra CM. Acquired syphilis in adults. N Engl J Med 1992;326:1060-1069.

3. Korean Centers for Disease Control and Prevention. Disease web statics system: syphilis [Internet]. Osong: Korean Centers for Disease Control and Prevention; c2002 [cited 2014 Mar 2]. Available from: http:// is.cdc.go.kr/nstat/index.jsp/.
4. Souza Varella Frazão M, Guimarães Vilaça T, Olavo Aragão Andrade Carneiro F, et al. Endoscopic aspects of gastric syphilis. Case Rep Med 2012;2012:646525.

5. Hunte W, al-Ghraoui F, Cohen RJ. Secondary syphilis and the nephrotic syndrome. J Am Soc Nephrol 1993;3:1351-1355.

6. Handoko ML, Duijvestein M, Scheepstra CG, de Fijter CW. Syphilis: a reversible cause of nephrotic syndrome. BMJ Case Rep 2013;2013:pii: bcr2012008279.

7. Fujisaki T, Tatewaki M, Fujisaki J. A case of gastric syphilis. Clin Gastroenterol Hepatol 2008;6:A34.

8. Greenstein DB, Wilcox CM, Schwartz DA. Gastric syphilis. Report of seven cases and review of the literature. J Clin Gastroenterol 1994;18:4-9.

9. Anai H, Okada Y, Okubo K, et al. Gastric syphilis simulating linitis plastica type of gastric cancer. Gastrointest Endosc 1990;36:624-626.

10. Abdu RA, Carter K, Pomidor WJ. Gastric syphilis mimicking linitis plastica. Arch Surg 1993;128:103-104.

11. Winters HA, Notar-Francesco V, Bromberg K, et al. Gastric syphilis: five recent cases and a review of the literature. Ann Intern Med 1992;116: 314-319.

12. Tang AL, Thin RN, Croft DN. Nephrotic syndrome and hepatitis in early syphilis. Postgrad Med J 1989;65:14-15.

13. Kwon HH, Woo CM, Oh HJ, et al. Nephrotic syndrome, hepatitis and gastric involvement in secondary syphilis. Korean J Nephrol 2004;23: 152-157. 